Dear author,

Please note that changes made in the online proofing system will be added to the article before publication but are not reflected in this PDF.

We also ask that this file not be used for submitting corrections. 


\title{
Deformation of atomic models and their equivalent continuum counterparts using Eringen's two-phase local/nonlocal model
}

\author{
Meral Tuna ${ }^{\mathrm{a}, *}$, Mesut Kirca ${ }^{\mathrm{a}}$, Patrizia Trovalusci ${ }^{\mathrm{b}}$ \\ a Faculty of Mechanical Engineering, Istanbul Technical University, Istanbul, Turkey \\ ${ }^{\mathrm{b}}$ Department of Structural and Geotechnical Engineering, Sapienza University of Rome, Via A. Gramsci, 53, Rome, 00197 Italy
}

\section{A R T I C L E I N F O}

\section{Article history:}

Received 15 December 2018

Revised 20 February 2019

Accepted 6 April 2019

Available online $\mathrm{xxx}$

\section{Keywords:}

Two-phase local/nonlocal model

Eringen

Atomic array

Finite element method

\begin{abstract}
A B S T R A C T
The aim of this contribution is to formulate equivalent continuum finite element model for twodimensional atomic arrays under plane-stress condition, based on Eringen's two phase local/nonlocal model. The interaction between the atoms is modelled using translational and rotational linear elastic springs including both nearest and second nearest neighbor relations. Explicit relations between those set of springs and material properties of associated continuum model is looked for by means of equivalency of potential energy stored in atomic bonds and strain energy of continuum. Possibility of reducing computational burden of full atomic models by equivalent continuum models is discussed. This study may be regarded as the first step in composing a partitioned-domain multiscale model; with possibly smoother transition between coarse and fine scales due to the ability of nonlocal continuum model in incorporating long-range interactions.
\end{abstract}

(C) 2019 Published by Elsevier Ltd.

\section{Introduction}

In recent years, continuum theories, capable of including the size effects, have gained considerable attention of researchers for the investigation of nano and micro sized structures. Among them, Eringen's constitutive model is one of the most widely used non-local theories, as it incorporates a small-scale parameter into the constitutive equation to capture micro/nano structural effects in continuous media [1-4]. It is originally formulated in an integral form, and simplified to a differential counterpart; yet, due to paradoxical outcomes of the latter for certain mechanical problems [5-7], the former form has also been substantially used [8-13].

This study concentrates on integral form of Eringen's two-phase local/nonlocal model [14] to investigate the behaviour of specific discrete systems. In fact, linking discrete systems to continuum approaches dates back to early molecular models of 19th century, through which the first attempts to derive the constitutive equations of continua was initiated $[15,16]$. These approaches are still quite promising in adopting discrete to scale dependent continuous models [17-19]. Here, particularly simple discrete system: 1-

This work was done when Meral Tuna was Visiting Researcher at DISG, Sapienza University of Rome, the support of which is gratefully acknowledged. The authors wish to thank Ugurcan Eroglu, visiting researcher at Sapienza University of Rome, for his assistance on formatting the manuscript.

* Corresponding author.

E-mail address: tunamer@itu.edu.tr (M. Tuna).
D atomic chain and 2-D atomic array models are considered. Interatomic potentials are modelled using linear elastic translational and rotational springs, for both the nearest, and second nearest neighbor relations from which the nonlocality arises. A linear system of equations for the atomic models are derived by means of the principle of minimimum total potential. Finite element formulation of continuum is developed for bar and plane stress problems, which paves the way to look for equivalent models by linking the strain energy on the continuum level to the energy stored in atomic bonds, assuming uniform deformation field [20-23], for 1-D and 2-D atomic structures, respectively. Indeed, explicit relations of material properties are obtained; albeit some of which require numerical integration schemes. Their equivalency are tested by examining same mechanical problem with both approaches. Despite some studies focusing on only translational springs and local elasticity [24,25], to the best of authorsâ knowledge this study is the first attempt to provide the closed-form expressions for nonlocal material properties in terms of spring constants introducing all possible interatomic relations. It is thought that the results provided herein are encouraging for the possibility of modelling transition zones of partitioned-domain multiscale models; as they indicate that Eringen's two phase model can capture the displacements of atomic arrays once the material properties ensuring energy equivalency are used. 


\section{Material and methods}

In this section, derivation of governing equation for static problems of 1-D atomic chain and 2-D atomic arrays, and FEM formulation of continuum with Eringen's two-phase local/nonlocal constitutive relation are presented. Distribution of the atoms/nodes are considered to be uniform throughout the axes. The nodes and the atoms in continuum and atomic models, respectively, do not necessarily need to be coincident. In fact, it is aimed to discretize the continuum models by using very coarse mesh in order to highlight its superiority in terms of computational cost. Materials in the continuum models are assumed to be linear, elastic and isotropic, in agreement with the atomic models which consist of identical atoms connected via linear elastic springs. Small deformations and displacements of all structures are considered; hence, linear models are used. For calculations, an in-house code is developed.

\subsection{One-dimensional case}

In 1-D case, both atomic and continuum models has one degree of freedom: translation in longitudinal axis $x$. In fact, it may not be of interest in practical applications; however, it is intended to examine them for the validation of present approach and integrity.

\subsubsection{Atomic chain}

1-D atomic chain model is composed by equally spaced (with a distance of $l_{a}$ ), identical atoms. Interatomic potential is represented using linear elastic translational springs representing both the nearest $\left(k_{1}\right)$ and the second nearest $\left(k_{2}\right)$ neighbor relations. Energy of $i$ th atom (i.e. $\mathcal{E}_{i}^{\text {atom }}$ ) with displacement of $u_{i}$ can be written as follows:

$$
\begin{aligned}
\mathcal{E}_{i}^{\text {atom }}= & \frac{k_{1}}{4}\left[\left(u_{i+1}-u_{i}\right)^{2}+\left(u_{i}-u_{i-1}\right)^{2}\right] \\
& +\frac{k_{2}}{4}\left[\left(u_{i+2}-u_{i}\right)^{2}+\left(u_{i}-u_{i-2}\right)^{2}\right]
\end{aligned}
$$

Remark 1. Eq. (1) is valid only for the atoms located at least $2 l_{a}$ away from the boundaries for our case in which interactions of atoms up to their second neighbors are taken into account. Hence, Eq. (1) must be simplified for the boundary atoms regarding the non-existing bonds.

Consequently, the internal energy of the atomic chain consisting of $N$ atoms can be expressed as

$\mathcal{E}_{\text {int }}^{a}=\sum_{i=1}^{N} \mathcal{E}_{i}^{a t o m}$

Each atom must be in equilibrium under the internal forces, $\left(f_{i}\right)_{\text {int }}$, and external forces, $\left(f_{i}\right)_{\text {ext }}$ :

$$
\begin{aligned}
& \left(\mathrm{f}_{i}\right)_{\mathrm{int}}+\left(\mathrm{f}_{i}\right)_{\mathrm{ext}}=0, \quad\left(\mathrm{f}_{i}\right)_{\mathrm{int}}=-\frac{\partial \mathcal{E}_{\text {int }}^{a}}{\partial u_{i}} \\
& =-\frac{\partial\left(\mathcal{E}_{i-2}^{a}+\mathcal{E}_{i-1}^{a}+\mathcal{E}_{i}^{a}+\mathcal{E}_{i+1}^{a}+\mathcal{E}_{i+2}^{a}\right)}{\partial u_{i}} .
\end{aligned}
$$

(3) provides a formulation similar to classical FEM:

$$
\mathbf{K}_{a} \mathbf{d}_{a}=\mathbf{f}_{a}
$$

where $\mathbf{f}_{a}, \mathbf{d}_{a}$ and $\mathbf{K}_{a}$ refer to external force vector, displacement vector, and the stiffness matrix, respectively.

\subsubsection{Continuum model}

From continuum mechanics point of view, 1-D atomic chain can be modelled as a bar structure characterized by a total length $L$, cross-sectional area, $A$, elasticity modulus $E$, and a material parameter $\kappa$ providing the nonlocal small-size effects through a kernel function, $\tau(r, \kappa)$. For a bar along a horizontal axis $x \in[0, L]$, the constitutive relation of Eringen's two-phase local/nonlocal model is,

$\sigma_{x}=\lambda D \varepsilon_{x}(x)+\psi \int_{0}^{L} \tau(|x-\bar{x}|, \kappa) D \varepsilon_{x}(\bar{x}) d \bar{x}$

where $\sigma_{x}$ and $\varepsilon_{x}$ keep their usual definitions of normal stress and strain. The weights of the local and nonlocal parts are regulated through a fraction coefficient, $\lambda \in[0,1]$, and $\psi=1-\lambda . \lambda=0$ and $\lambda=1$ induce full nonlocal and full local models, respectively. Being different from local elasticity, in nonlocal models, stress at a point is linked to the strain of the entire domain through a kernel function, which is assumed bi-exponential

$\tau(r, \kappa)=e^{-\frac{r}{\kappa}} /\left(2 \pi^{n-1} \kappa^{n}\right)$

$n$ being the dimension of the structure. Other kernel functions to represent nonlocal effects are also available [26].

In natural coordinate system $\zeta=\left(l_{c}+2 x-2 x_{2}\right) / l_{c}$, displacement field may be approximated by two-noded linear bar elements with equal length, $l_{c}$.

$$
\begin{aligned}
\mathbf{u}_{e}(\zeta) & =\mathbf{N} \mathbf{d}_{e}, \quad \mathbf{N}(\zeta)=\left[\frac{(1-\zeta)}{2} \frac{(1+\zeta)}{2}\right] \\
\mathbf{d}_{e} & =\left\{d_{1_{x}} d_{2_{x}}\right\}_{e}^{T}, \quad \varepsilon_{e}=\mathbf{B}_{e} \mathbf{d}_{e} \\
\mathbf{B}_{e} & =\frac{\partial \mathbf{N}(\zeta)}{\partial \zeta} \frac{\partial \zeta}{\partial x}=\left[-\frac{1}{2} \frac{1}{2}\right] \mathbf{J}_{e}^{-1}
\end{aligned}
$$

with $d_{1 x}$ and $d_{2 x}$ being longitudinal displacement of the 1 st and the 2 nd node, and $\varepsilon_{e}$ the strain of element $e$. With those definitions at hand, strain energy of the bar,

$U=\frac{1}{2} \int_{V} \varepsilon(x) \sigma(x) d V(x)$

With $L_{m n}=\mathbf{d}_{m}^{T} \mathbf{B}_{m}^{T} E_{n} \mathbf{B}_{n} \mathbf{d}_{n}$, it may be approximated, by using $M$ uniform elements, as

$$
\begin{aligned}
U_{F E M}= & \frac{A}{2}\left(\lambda \sum_{m=1}^{M} \int_{-1}^{1} L_{m m} \operatorname{det}\left|\mathbf{J}_{m}\right| \mathrm{d} \zeta\right. \\
& \left.+\psi \sum_{m=1}^{M} \sum_{n=1}^{M} \int_{-1}^{1} \int_{-1}^{1} \frac{e^{\frac{|\zeta-\bar{\xi}|}{2 \kappa}}}{2 \kappa} L_{m n} \operatorname{det}\left|\mathbf{J}_{n}\right| \operatorname{det}\left|\mathbf{J}_{m}\right| \mathrm{d} \bar{\zeta} \mathrm{d} \zeta\right)
\end{aligned}
$$

Total potential, $\Pi$, can be written in terms of strain energy with 108 FEM approach, $U_{\text {FEM }}$, plus external work potential, $W_{\text {FEM }}$, and must be minimum for equilibrium; hence,

$$
\Pi=U_{F E M}+W_{F E M}, \quad \frac{\partial \Pi}{\partial \mathbf{d}_{i}}=0,(i=1,2, \ldots, M)
$$

which requires, $\mathbf{d}$ being the global displacement vector. Then, inserting (7) into $(10)_{2}$ provides

$$
\begin{aligned}
\mathbf{f}_{m} & =\lambda \mathbf{k}_{m} \mathbf{d}_{m}+\psi \mathbf{k}_{m m} \mathbf{d}_{m}+2 \psi \sum_{n=1, n \neq m}^{M} \mathbf{k}_{m n} \mathbf{d}_{n} \\
\mathbf{k}_{m} & =\frac{E A}{l_{c}}\left[\begin{array}{cc}
1 & -1 \\
-1 & 1
\end{array}\right] \\
\mathbf{k}_{m m} & =\frac{E A\left(l_{c}+\kappa\left(e^{-\frac{l_{c}}{\kappa}}-1\right)\right)}{l_{c}^{2}}\left[\begin{array}{cc}
1 & -1 \\
-1 & 1
\end{array}\right] \\
\mathbf{k}_{m n} & =\frac{\kappa E A e^{-\frac{(|m-n|+1) l_{c}}{\kappa}}\left(e^{\frac{l_{c}}{\kappa}}-1\right)^{2}}{4 l_{c}^{2}}\left[\begin{array}{cc}
1 & -1 \\
-1 & 1
\end{array}\right]
\end{aligned}
$$

$\mathbf{k}_{m m}$, stands for the contribution of the $m$ th element to its own energy, while $\mathbf{k}_{m n}$ and $\mathbf{k}_{n m}$ account for the influence exerted on the $m$ th element by the remaining elements, and the influence exerted by the $m$ th element to the other elements. Also, it is vital to 

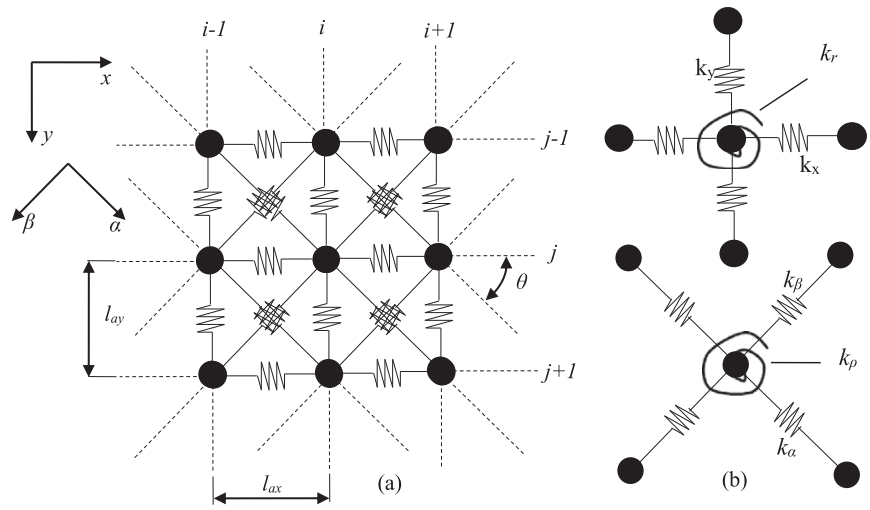

Fig. 1. Illustration of 2-D atomic array model.

point out that $\mathbf{k}_{m n}=\mathbf{k}_{n m}^{T}$ in case of homogeneous material properties. Consequently, global form of the stiffness matrix including all degrees of freedom is constructed through proper assemblage of the element stiffness matrices. Based on the minimum potential energy principle, global form of finite element formulation can be represented as:

$\mathbf{K}_{c} \mathbf{d}_{c}=\mathbf{f}_{c}$

where $\mathbf{f}_{c}$ and $\mathbf{d}_{c}$ are external force and displacement vector, respectively, and $\mathbf{K}_{c}$ is called as the global stiffness matrix with a dimension of $(M+1) \times(M+1)$. To validate FE model, the results are compared with the analytical expressions given in [27] for the same boundary conditions. Although slight differences at the boundaries due to different solution techniques, a very good agreement is achieved.

Finally, regarding the energy equivalency for the uniform deformation field of atomic and continuum models, following relation between the material properties and the spring constants is obtained.

$k_{1}+4 k_{2}=\frac{E A}{l_{a}}\left[\psi\left(1+\frac{\kappa}{l_{c}} g(z n)\right)+\lambda\right]$

$g(z n)=\left(e^{-\frac{(z n+1) l_{c}}{\kappa}}-e^{-\frac{z n l_{c}}{\kappa}}\right)$

where $z n$ refers to the total number of elements that fall in the radius of influence zone of an element. Hence, its value should be increased with the nonlocality.

\subsection{Two-dimensional case}

To parameterize the position of points, components of forces and displacements, etc., a Cartesian coordinate system $x, y$ with unit vectors $\mathbf{e}_{1}, \mathbf{e}_{2}$ are used. In 2-D case, atomic and continuum models possess two translational degree of freedoms: planar displacements, $u$ and $v$; the components of displacement vector $\mathbf{u}$.

\subsubsection{Atomic array}

In the present study, a square 2-D atomic array model is composed using equally spaced (i.e. $l_{a x}=l_{a y}=l_{a}$ ), identical atoms as illustrated in Fig. 1a. Linear elastic translational and rotational springs for the nearest and the second nearest neighbor relations are utilized to represent the atomic interactions. The translational springs with constants $k_{x}, k_{y}, k_{\alpha}, k_{\beta}$, are oriented along $x, y, \alpha$ and $\beta$ directions, respectively, while the rotational springs with constants $k_{r}$ and $k_{\rho}$ connect the atoms positioned along $x-y$ and $\alpha-\beta$ axes, accordingly (see Fig. 1b).

The internal energy can be expressed in terms of bond length and bond angle variations by specifying each contribution individually. For brevity, instead of the energy of the atom, contribution

of the rotational springs located at the $j$ th row and $i$ th column of 156 the array is given below:

$$
\begin{aligned}
& \left(\mathcal{E}_{j i}^{\text {bond }}\right)_{\text {rot }}= \\
& \left.\sum_{n=1}^{2}\left\{\begin{array}{l}
k_{r_{n}} \\
+\left(\frac{u_{j-n, i}-u_{j, i}}{l_{a y}}+\frac{v_{j, i-n}-v_{j, i}}{l_{a x}}\right)^{2} \\
+\left(\frac{u_{j, i}-u_{j+n, i}}{l_{a y}}+\frac{v_{j, i}-v_{j, i+n}}{l_{a x}}\right)^{2} \\
+\left(\frac{u_{j, i}-u_{j-n, i}}{l_{a y}}+\frac{v_{j, i+n}-v_{j, i}}{l_{a x}}\right)^{2}
\end{array}\right]\right) \\
& \left.+\frac{k_{\rho_{n}}}{2 \ell^{2}}\left(\begin{array}{l}
{\left[\left(\left\{u_{j, i}-u_{j+n, i-n}+v_{j, i}-v_{j+n, i+n}\right\} c\right.\right.} \\
\left.+\left\{u_{j+n, i+n}-u_{j, i}+v_{j, i}-v_{j+n, i-n}\right\} s\right)^{2} \\
\left(\left\{u_{j+n, i-n}-u_{j, i}+v_{j, i}-v_{j-n, i-n}\right\} c\right. \\
\left.+\left\{u_{j-n, i-n}-u_{j, i}+v_{j+n, i-n}-v_{j, i}\right\} s\right)^{2} \\
+\left(\left\{u_{j, i}-u_{j-n, i+n}+v_{j+n, i+n}-v_{j, i}\right\} c\right. \\
\left.+\left\{u_{j, i}-u_{j+n, i+n}+v_{j, i}-v_{j-n, i+n}\right\} s\right)^{2} \\
+\left(\left\{u_{j-n, i+n}-u_{j, i}+v_{j-n, i-n}-v_{j, i}\right\} c\right. \\
\left.+\left\{u_{j, i}-u_{j-n, i-n}+v_{j-n, i+n}-v_{j, i}\right\} s\right)^{2}
\end{array}\right]\right\}
\end{aligned}
$$

where $s$ and $c$ are $\sin \theta$ and $\cos \theta$, respectively, the subscripts 1 and 2 of $k$ correspond to the nearest and second-nearest relations, and $\ell^{2}=l_{a x}^{2}+l_{a y}^{2}$. Energy of an atom due to translations is

158 $\left(\mathcal{E}_{j, i}^{\text {atom }}\right)$

$=\sum_{n=1}^{2}\left\{\frac{k_{x_{n}}}{4}\left[\left(u_{j, i+n}-u_{j, i}\right)^{2}+\left(u_{j, i}-u_{j, i-n}\right)^{2}\right]\right.$

$+\frac{k_{y_{n}}}{4}\left[\left(v_{j+n, i}-v_{j, i}\right)^{2}+\left(v_{j, i}-v_{j-n, i}\right)^{2}\right]$

$+\frac{k_{\alpha_{n}}}{4}\left[\left(\left\{u_{j+n, i+n}-u_{j, i}\right\} c+\left\{v_{j+n, i+n}-v_{j, i}\right\} s\right)^{2}\right.$

$\left.+\left(\left\{u_{j, i}-u_{j-n, i-n}\right\} c+\left\{v_{j, i}-v_{j-n, i-n}\right\} s\right)^{2}\right]$

$+\frac{k_{\beta_{n}}}{4}\left[\left(\left\{u_{j, i}-u_{j+n, i-n}\right\} s+\left\{v_{j+n, i-n}-v_{j, i}\right\} c\right)^{2}\right.$

$\left.\left.+\left(\left\{u_{j-n, i+n}-u_{j, i}\right\} s+\left\{v_{j, i}-v_{j-n, i+n}\right\} c\right)^{2}\right]\right\}$

Eventually, total internal energy of a 2-D array of $N$ atoms, is calculated as follows.

$\mathcal{E}_{\text {int }}^{a}=\sum_{j=1}^{N} \sum_{i=1}^{N}\left(\mathcal{E}_{j, i}^{a t o m}\right)_{\text {str }}+\sum_{j=1}^{N} \sum_{i=1}^{N}\left(\mathcal{E}_{j, i}^{\text {bond }}\right)_{\text {rot }}$

Note that the expressions given in Eqs. (14) and (15) are valid only for the atoms and the bonds located sufficiently away from the boundaries. For the others, some simplifications are required to avoid miscalculation.

Equlibrium condition of 2-D array requires

$\left(\mathbf{f}_{i}\right)_{\text {int }}+\left(\mathbf{f}_{i}\right)_{\mathrm{ext}}=0, \quad\left(\mathbf{f}_{i}\right)_{\text {int }}=-\frac{\partial \mathcal{E}_{\text {int }}^{a}}{\partial \mathbf{u}_{i}}$

which may be represented in an identical form to (4), where the components of stiffness matrix are given as,

$K_{2 i-1,2 j-1}=\frac{\partial \mathcal{E}_{\text {int }}^{a}}{\partial u_{i} \partial u_{j}}, \quad K_{2 i, 2 j}=\frac{\partial \mathcal{E}_{\text {int }}^{a}}{\partial v_{i} \partial v_{j}}$

\subsubsection{Continuum model}

From the view of continuum mechanics, 2-D atomic array can be modelled a continuum occupying 2-D planar region. Constitu- 
tive relation is similar to what is assumed in 1-D case:

$\boldsymbol{\sigma}(x, y)=\lambda \mathbf{C}: \boldsymbol{\varepsilon}(x, y)+\psi \iint \tau(r, \kappa) \mathbf{C}: \boldsymbol{\varepsilon}(\bar{x}, \bar{y}) d \bar{A}$

where, in case of plane-stress condition,

$$
\begin{gathered}
\boldsymbol{\sigma}(x, y)=\left\{\begin{array}{l}
\sigma_{x x} \\
\sigma_{y y} \\
\sigma_{x y}
\end{array}\right\}, \quad \boldsymbol{\varepsilon}(x, y)=\left\{\begin{array}{l}
\varepsilon_{x x} \\
\varepsilon_{y y} \\
2 \varepsilon_{x y}
\end{array}\right\}, \\
\mathbf{C}=\frac{E}{\left(1-v^{2}\right)}\left[\begin{array}{lll}
1 & v & 0 \\
v & 1 & 0 \\
0 & 0 & \frac{(1-v)}{2}
\end{array}\right]
\end{gathered}
$$

$d \bar{A}$ is equal to $d \bar{x} d \bar{y}$, differential area element. Kernel function $\tau(r, \kappa)$ is given in (6), obviously for $n=2$ [28], and $r$ is the Euclidean distance between the point of interest and its neighbor points.

For finite element (FE) approximation to displacement and strain fields within an element $e ; \mathbf{u}_{e}$ and $\boldsymbol{\varepsilon}_{e}$, discretization with 4-noded linear elements with bilinear shape functions (i.e. $\mathbf{N}$ ) are used. In a natural coordinate system:

$u_{e}(\zeta, \eta)=N(\zeta, \eta) d_{e}$

$$
\boldsymbol{\varepsilon}_{e}=\mathbf{L}_{e} \mathbf{N} \mathbf{d}_{e}=\mathbf{B}_{e} \mathbf{d}_{e}
$$

$\mathbf{L}_{e}=\left[\begin{array}{cc}\frac{\partial}{\partial x} & 0 \\ 0 & \frac{\partial}{\partial y} \\ \frac{\partial}{\partial y} & \frac{\partial}{\partial x}\end{array}\right], \quad \mathbf{J}=\frac{1}{2}\left[\begin{array}{cc}l_{c x} & 0 \\ 0 & l_{c y}\end{array}\right]$

The strain energy of a plate with thickness $h$ is known as,

$$
\begin{aligned}
U= & \frac{h}{2}\left(\lambda \iint \boldsymbol{\varepsilon}(x, y)^{T} \mathbf{C} \boldsymbol{\varepsilon}(x, y) d A\right. \\
& \left.+\psi \iiint \int \tau(r, \kappa) \boldsymbol{\varepsilon}^{T}(x, y) \overline{\mathbf{C}} \boldsymbol{\varepsilon}(\bar{x}, \bar{y}) d \bar{A} d A\right)
\end{aligned}
$$

Then, the principle of minimum total potential, similar to what is done in 1-D case, provides the FE formulation of 2-D continua,

$$
\begin{aligned}
\mathbf{f}_{m} & =\lambda \mathbf{k}_{m} \mathbf{d}_{m}+\psi \mathbf{k}_{m m} \mathbf{d}_{m}+2 \psi \sum_{n=1, n \neq m}^{M} \mathbf{k}_{m n} \mathbf{d}_{n}, \\
\mathbf{k}_{m} & =h \int_{-1}^{1} \int_{-1}^{1} \mathbf{B}_{m}^{T}(\zeta, \eta) \mathbf{C}_{m} \mathbf{B}_{m}(\zeta, \eta) \operatorname{det}\left|\mathbf{J}_{m}\right| \mathrm{d} \zeta \mathrm{d} \eta, \\
\mathbf{k}_{m n} & =\frac{h}{2} \int_{-1}^{1} \int_{-1}^{1} \int_{-1}^{1} \int_{-1}^{1} \tau(r, \kappa) \mathbf{A}_{m n} \mathrm{~d} \bar{\zeta} \mathrm{d} \bar{\eta} \mathrm{d} \zeta \mathrm{d} \eta, \\
r & =\sqrt{(\zeta-\bar{\zeta})^{2}+(\eta-\bar{\eta})^{2}}, \\
\mathbf{A}_{m n} & =\mathbf{B}_{m}^{T}(\zeta, \eta) \mathbf{C}_{n} \mathbf{B}_{n}(\bar{\zeta}, \bar{\eta}) \operatorname{det}\left|\overline{\mathbf{J}}_{n}\right| \operatorname{det}\left|\mathbf{J}_{m}\right| .
\end{aligned}
$$

In terms of global force vector $\mathbf{f}_{c}$, global displacement vector $\mathbf{d}_{c}$ and global stiffness matrix $\mathbf{K}_{c}$, an identical representation of (23) to (12) is possible.

Remark 2. The integration operations of the nonlocal part are per-

\begin{tabular}{|c|c|c|}
\hline case & $\lambda$ & $\kappa[\mathrm{nm}]$ \\
\hline 1 & 1 & any value \\
\hline 2 & 0.2 & $0.025 L$ \\
\hline 3 & 0.2 & $0.050 \mathrm{~L}$ \\
\hline 4 & 0.7 & $0.050 \mathrm{~L}$ \\
\hline
\end{tabular}
formed using Gauss Quadrature (GQ) method. The number of GQ points should be increased depending on the ratio between the nonlocal parameter and the element length (i.e. $\kappa / l_{c x}$ or $\kappa / l_{c y}$ ), and the calculated part of the stiffness matrix (i.e. $k_{m m}$ or $k_{m n}$ ).

Despite its capabilities, integral form of nonlocal theory based FEM formulation has been only conducted by a limited number of researchers, such as [29-31]. For the validation of the FE model, the results are compared with the ones given in [30] considering the same boundary conditions. Slight differences in the strain field is encountered only at the boundaries of the domain due to different element types: in the reference article 8-noded Serendipity element is used, while in the present study the formulation is derived based on the 4-noded linear elements. In order to obtain material properties in terms of spring constants (i.e. $E$ and $\kappa$ ), the total energy of the unit cell in the atomic model and the total energy of the corresponding element in the continuum model are compared under uniform deformation fields such as; uniaxial, biaxial, pure shear and simple shear. Considering isotropy,

$k_{x}=k_{y}=k_{l}, \quad k_{\alpha}=k_{\beta}=k_{s}, \quad G=\frac{E}{2(1+v)}$

200 201 202 203 204 205 206 207 208

following expressions are obtained:

$$
\begin{aligned}
E & =\frac{1}{\lambda+\psi \xi} \frac{\left(k_{E_{1}}+2 k_{E_{2}}\right)\left(8 k_{E_{3}}+k_{E_{1}} l_{a}^{2}\right)}{h\left(4 k_{E_{3}}+\left(k_{E_{1}}+k_{E_{2}}\right) l_{a}^{2}\right)} \\
v & =\frac{-4 k_{E_{3}}+k_{E_{2}} l_{a}^{2}}{4 k_{E_{3}}+\left(k_{E_{1}}+2 k_{E_{2}}\right) l_{a}^{2}}, \quad G=\frac{1}{\lambda+\psi \xi} \frac{4 k_{E_{4}}+k_{E_{2}} l_{a}^{2}}{h l_{a}^{2}}, \\
k_{r} & =k_{\rho}+\left(\frac{k_{l}}{8}-\frac{k_{s}}{4}\right) l_{a}^{2} \\
k_{E_{1}} & =k_{l_{1}}+4 k_{l_{2}}, \quad k_{E_{2}}=k_{s_{1}}+4 k_{s_{2}}, \\
k_{E_{3}} & =k_{\rho_{1}}+4 k_{\rho_{2}}, \quad k_{E_{4}}=k_{r_{1}}+4 k_{r_{2}},
\end{aligned}
$$

with $\xi$, which varies with the value of the nonlocal parameter, be- 210 ing basically a constant arising from numerical integration; hence, 211 it depends on $z n$ and the number of GQ points. An explicit expres- 212 sion for $\xi$ is possible, but too long to be reported within the length 213 of this article.

\section{Numerical examples}

In this section, static response of atomic and continuum models are examined for some benchmark problems of practical importance.

\subsection{One-dimensional case}

An atomic chain of $L=1[\mathrm{~nm}]$ length, which is thought of consisting 161 atoms, is investigated as an example. 20 finite elements are used to construct its approximated continuum model. The spring constants stimulating the interaction with the nearest and the second nearest neighbor atoms are assumed equal: $k_{1}=k_{2}=80[\mathrm{nN} / \mathrm{nm}$ ]. Young's modulus is calculated by Eq. (25), assuming $A=1\left[\mathrm{~nm}^{2}\right]$. The structure is investigated under both constant and linearly varying normal force, which may be due to a tip point load, $f=1 \mathrm{nN}$, and uniformly distributed load $q=f / L$. Material properties are provided in Table 1. Number of elements inside the radius of influence zone, $z n$, is set to 9 considering highest $\kappa=0.05 \mathrm{~L}[\mathrm{~nm}]$. Assuming a fixed mid-point, axial displacement fields of atomic and equivalent continuum models are plotted in Fig. 2, where an excellent agreement is achieved for all cases. Only appreciable discrepancy is apparent for uniform strain field around boundaries of the domain; see Fig. 2a. This is due to the phenomenon known as boundary effect, as also pointed out by Remark 1. However, it also depends on the state of stress around the corresponding boundary domain. This is also evidenced by 


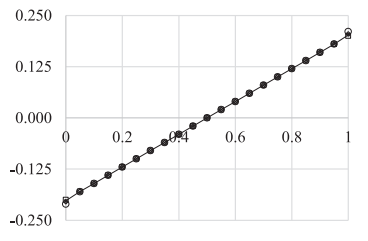

(a)

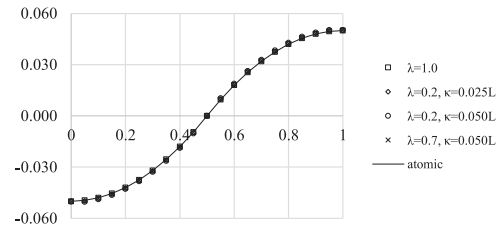

(b)

Fig. 2. Displacement values at atoms/nodes under (a) tip point load $f$, and (b) equally distributed axial load, $q . x$ and $y$ axis denote coordinate $x[\mathrm{~nm}]$ and axial displacement $u[\mathrm{~nm}]$, respectively.

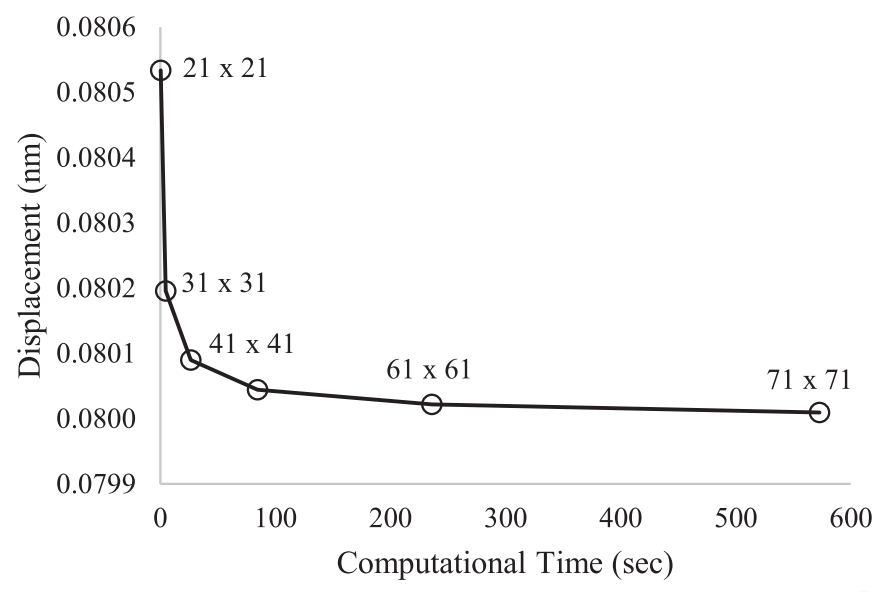

Fig. 3. The variation of $u(40 \mathrm{~nm}, 50 \mathrm{~nm})$ and computational time with respect to total number of atoms.

deformation field of uniformly distributed load in which case, a perfect correspondence between displacement is achieved, as illustrated at Fig 2 b. Nevertheless, minimizing such a discrepancy looks possible by proper selection of nonlocal material parameters.

\subsection{Two-dimensional case}

For 2-D case, an evenly spaced atomic array occupying a square region with edge length $L=100[\mathrm{~nm}]$ is considered. Continuum approximation of it consists of $21 \times 21$ nodes, while different number of atoms are considered. Similar to 1-D problem, spring constants are assumed equal.

There are two important points to be stressed out:

(1) Spring constants can be arbitrarily determined as long as they satisfy Eqs. (24) and (25) 4 .

(2) Different values of spring constants may yield same material properties as clearly seen from Eq. (25).

Regarding these facts, although an infinite number of alternatives exists, only the followings are considered: $k_{l}=1.5$ $[\mathrm{nN} / \mathrm{nm}], k_{s}=0.91667[\mathrm{nN} / \mathrm{nm}], k_{\rho}=0.0625 l_{a}^{2}[\mathrm{nN} . \mathrm{nm}]$ and $k_{r}=$ $0.0208333 l_{a}^{2}$ [nN.nm]. Numerical experiments showed that consideration of different values for spring constants yield practically identical deformation fields as the number of atoms are increased. However, for the sake of brevity, results of those numerical experiments paving the way to this conclusion are not reported here.

As a first step, the deformation fields of the atomic array with different number of atoms are investigated. Fig. 3 shows the displacement along $x$-axis at a point and the computational time required, for the different number of atoms, in case of uniaxial loading. In the light of Fig. 3, $61 \times 61$ number of atoms looks to be an optimum choice considering the convergence and computational burden.

For continuum model, material properties are listed in Table 1. Poisson's ratio is calculated as 0.25 for all cases as it does not depend on either nonlocal parameter or fraction coefficient, while

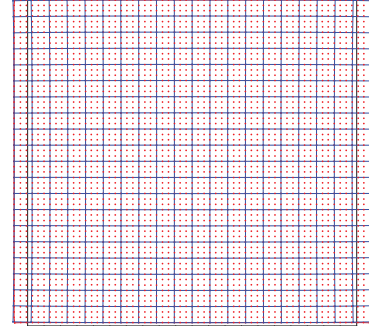

(a)

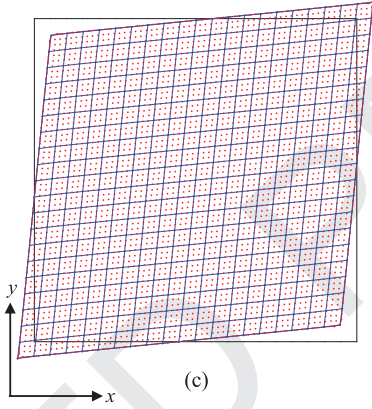

Fig. 4. Deformed configurations of atomic (red), and continuum (blue) models under (a) uniaxial, (b) biaxial, (c) pure shear, and (d) point load conditions. (Black: undeformed configuration) (For interpretation of the references to colour in this figure legend, the reader is referred to the web version of this article.)

Youngâs modulus is obtained assuming $h=1[\mathrm{~nm}]$. Following loading cases are investigated:

(a) constant distributed uniaxial load :

$q_{x}(0, y)=-q_{x}(L, y)=-f / L$,

(b) constant distributed biaxial load:

$q_{x}(0, y)=q_{y}(x, 0)=-f / L$,

$q_{x}(L, y)=q_{y}(x, L)=f / L$

(c) constant distributed shear load:

$q_{x}(x, 0)=f / L, q_{x}(x, L)=-f / L$,

$q_{y}(0, y)=f / L, q_{y}(L, y)=-f / L$

(d) point load applied to midpoint of upper edge :

$f_{p}(L / 2, L)=-f / 10$,

where $f=100[\mathrm{nN}]$. For the first three loading conditions, the center point of the domain is assumed fixed. For the last one, the displacement of atoms/nodes located at the bottom edge are restricted along $y$ direction only.

Mechanical problems considered in cases (a)-(c) are basically simple benchmark problems providing uniform strain fields. On the other hand, case (d) may be regarded as a coarse approximation to half infinite continuum under point load, also known as Flamant problem. It is simply examined to test the equivalent models wide range of deformation gradients to see its capability.

The deformed configurations of the atomic and continuum models with $\kappa=0.050 \mathrm{~L}$ and $\lambda=0.7$ are illustrated in Fig. 4 . At the first glance, a very good agreement in terms of the displacements are observed. More in detail, slight difference at boundaries, which are even more pronounced for corner points, are observed due to the discrete nature of atomic model, in addition to what is said for 1-D case. Obviously, including additional connections between atoms and/or considering different distributions of them will enrich the atomic model, which will eventually lead to a more similar behavior. Displacement fields for the first three loading cases exhibit a similar trend to atomic chain with tip point load, while case (d) deserves more attention. Vertical displacement of nodes/atoms at $y=L / 4,3 L / 4$ are illustrated explicitly at Fig. 5. As the deforma- 


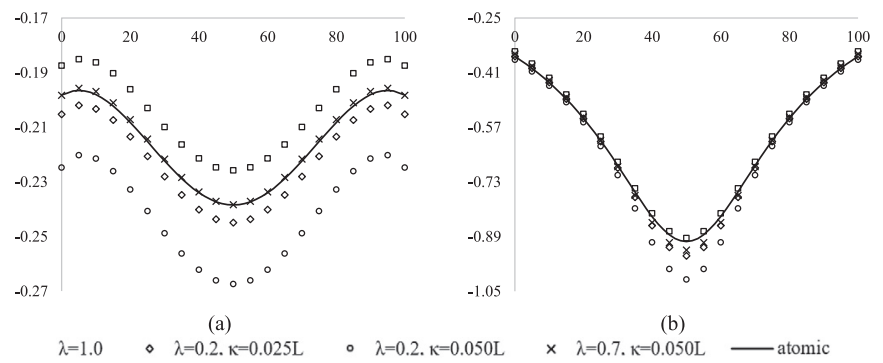

Fig. 5. Displacement values of atoms/nodes located at (a) $y=L / 4$ (b) $y=3 L / 4$ for case (d). $x$ and $y$ axis denote coordinate $x[\mathrm{~nm}]$ and vertical displacement $v[\mathrm{~nm}]$, respectively.

Table 2

Comparison of internal energies [nN.nm] of discrete, local continuum and nonlocal continuum models for 2-D problems.

\begin{tabular}{lllll}
\hline & $(\mathrm{a})$ & $(\mathrm{b})$ & $(\mathrm{c})$ & $(\mathrm{d})$ \\
\hline atomic & 823.6 & 1241.8 & 2116.5 & 39.443 \\
case 1 & 800.0 & 1200.0 & 2000.0 & 24.616 \\
case 4 & 832.1 & 1247.8 & 2081.0 & 29.014 \\
\hline
\end{tabular}
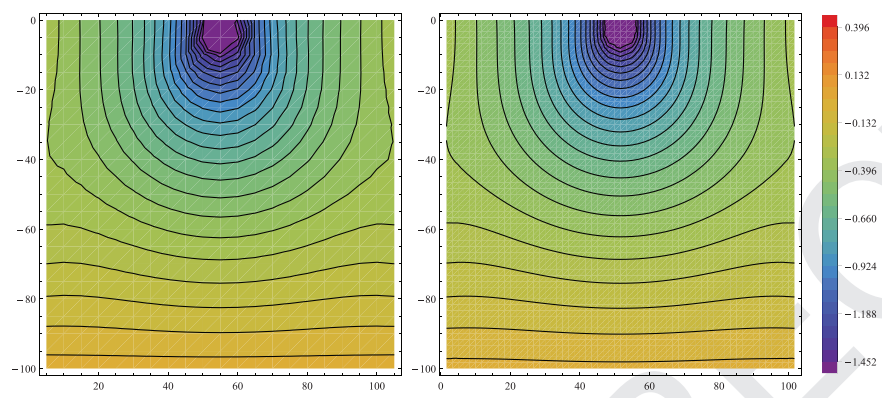

Fig. 6. Contour plots of vertical deformation fields for case (d). Left: Nonlocal continuum model (Case 4). Right: Atomic model.

tion gradients are appreciable, displacements of equivalent continuum models do not overlap with each other for arbitrary selection of material properties. On the other hand, suitable selection of them (e.g. $\lambda=0.7$, and $\kappa=0.05 L$ ) may provide a good match in terms of not only displacement fields, but also total internal energy (see Table 2). Another important interpretation of Fig. 5 may be the following: as Eringen's constitutive relation may be tailored to match atomic displacements, it may also be used in parititoneddomain multiscale models where a strong or weak compatibility of displacement field atomic and continuum models are looked for. The coherence between models are also pointed out through comparing the contour plots illustrated in Fig. 6, for case (d). As an inevitable outcome of finer discretization, the vertical displacement field of atomic model is slightly smoother than its continuum approximation.

\section{Conclusion}

Present study deals with equivalent continuum finite element models of 2-D atomic array based on Eringen's two phase local/nonlocal model. To have physically reasonable continuum approximation to atomic model, material properties of the former are obtained in closed-form, admitting an energy equivalency under uniform deformation. The advantage and capability of equivalent continuum nonlocal model are highlighted in terms of both accuracy and computational expense via comparing models under various loading scenarios. Numerical experiments show that even though the total number of degree of freedoms is reduced by $90 \%$, all continuum models are well capable of providing very accurate solutions, while it becomes dependent on nonlocal material parameters for increasing deformation gradients. Such a behaviour is expected due to analogous nature of nonlocal and atomic models, which may help recovering more accurate solutions. This could be further investigated by focusing on entire domain in case of general deformation fields, and might lead to additional constraints on nonlocal material properties, even to unique determination of them. Nevertheless, via exploiting the capability of continuum models including nonlocal effects, a smoother transition between atomic and continuum regions of a partitioned-domain multi-scale model is expected, which is the scope on an ongoing project.

\section{Supplementary material}

Supplementary material associated with this article can be found, in the online version, at doi:10.1016/j.mechrescom.2019.04. 004.

\section{References}

[1] J. Peddieson, G.R. Buchanan, R.P. McNitt, Application of nonlocal continuum models to nanotechnology, Int. J. Eng. Sci. 41 (2003) 305-312.

[2] C. Polizotto, Nonlocal elasticity and related variational principles, Int. J. Solids Struct. 38 (2001) 7359-7380.

[3] J.N. Reddy, Nonlocal theories for bending, buckling and vibration of beams, Int J. Eng. Sci. 45 (2007) 288-307.

[4] J.K. Phadikar, S.C. Pradhan, Variational formulation and finite element analysis for nonlocal elastic nanobeams and nanoplates, Comput. Mater. Sci. 49 (2010) 492-499.

[5] N. Challamel, C.M. Wang, The small length scale effect for a non-local cantilever beam: a paradox solved, Nanotechnology 19 (2008) 345703(7).

[6] J. Fernaández-Sáez, R. Zaera, J.A. Loya, J.N. Reddy, Bending of euler-bernoulli beams using Eringen's integral formulation: a paradox resolved, Int. J. Eng. Sci. 99 (2016) 107-116.

[7] C. Li, L. Yao, W. Chen, S. Li, Comments on nonlocal effects in nano-cantileve beams, Int. J. Eng. Sci. 87 (2015) 47-57.

[8] M. Tuna, M. Kirca, Exact solution of Eringen's nonlocal integral model for vibration and buckling of euler-bernoulli beam, Int. J. Eng. Sci. 107 (2016) 54-67.

[9] M. Tuna, M. Kirca, Exact solution of Eringen's nonlocal integral model for bending of Eulerbernoulli and timoshenko beams, Int. J. Eng. Sci. 105 (2016) 80-92.

[10] J. Fernández-Sáez, R. Zaera, Vibrations of Bernoulli-euler beams using the twophase nonlocal elasticity theory, Int. J. Eng. Sci. 119 (2017) 232-248.

[11] X. Zhu, W. Yuanbin, D. Hui-Hui, Buckling analysis of Euler-Bernoulli beams using Eringens two-phase nonlocal model, Int. J. Eng. Sci. 116 (2017) 130-140.

[12] H.M. Numanoglu, B. Akgoz, O. Civalek, On dynamic analysis of nanorods, Int. J. Eng. Sci. 130 (2018) 33-50.

[13] C. Polizzotto, P. Fuschi, A. Pisano, A nonhomogeneous nonlocal elasticity model, Eur. J. Mech. 25 (2) (2006) 308-333.

[14] A.C. Eringen, Theory of nonlocal elasticity and some applications, Res. Mechan ica 21 (1987) 313-342.

[15] P. Trovalusci, D. Capecchi, G. Ruta, Genesis of the multiscale approach for materials with microstructure, Arch. Appl. Mech. 79 (11) (2008) 981.

[16] D. Capecchi, G. Ruta, P. Trovalusci, Voigt and poincaré's mechanistic-energetic approaches to linear elasticity and suggestions for multiscale modelling, Arch. Appl. Mech. 81 (11) (2011) 1573-1584.

[17] P. Trovalusci, Molecular Approaches for Multifield Continua: Origins and Current Developments, Springer, Vienna, pp. 211-278.

[18] P. Trovalusci, A. Pau, Derivation of microstructured continua from lattice systems via principle of virtual works: the case of masonry-like materials as micropolar, second gradient and classical continua, Acta Mechanica 225 (1) (2014) 157-177.

[19] P. Trovalusci, Discrete to Scale-Dependent Continua for Complex Materials: A Generalized Voigt Approach Using the Virtual Power Equivalence, Springer International Publishing, Cham, pp. 109-131.

[20] E. Tadmor, R. Miller, Modeling Materials: Continuum, Atomistic and Multiscale Techniques, Cambridge University Press, 2011.

[21] J. Fan, Multiscale Analysis of Deformation and Failure of Materials, Microsys tem and Nanotechnology Series (ME20), Wiley, 2011.

[22] R. Ansari, A. Shahabodini, H. Rouhi, Prediction of the biaxial buckling and vibration behavior of graphene via a nonlocal atomistic-based plate theory, Compos. Struct. 95 (2013) 88-94.

[23] M. Al-Kharusi, K. Alzebdeh, T. Pervez, An atomistic-based continuum modeling for evaluation of effective elastic properties of single-walled carbon nanotubes, J. Nanomater. 8641954 (2016) 13pages.

[24] V. Baudet, M. Beuve, F. Jaillet, B. Shariat, F. Zara, New mass-spring system integrating elasticity parameters in 2d, 2007.

[25] P. Suryawanshi, A. Gupta, A novel mass spring model topology for modeling of linear isotropic homogenous materials, in: Proceedings of the ASME International Mechanical Engineering Congress and Exposition, 2015, p. 8pages.
335

\section{6}


[26] A.C. Eringen, On differential equations of nonlocal elasticity and solutions of screw dislocation and surface waves, J. Appl. Phys. 54 (9) (1983) 4703-4710.

[27] E. Benvenuti, A. Simone, One-dimensional nonlocal and gradient elasticity: closed-form solution and size effect, Mech. Res. Commun. 48 (2013) 46-51.

[28] S. Ghosh, V. Sundararaghavan, A.M. Waas, Construction of multi-dimensional isotropic kernels for nonlocal elasticity based on phonon dispersion data, Int. J. Solids Struct. 51 (2) (2014) 392-401.
[29] A.A. Pisano, A. Sofi, P. Fuschi, Finite element solutions for nonhomogeneous nonlocal elastic problems, Mech. Res. Commun. 36 (7) (2009) 755-761.

(2d finite element based

[31] P. Fuschi, A. Pisano, D. De Domenico, Plane stress problems in nonlocal elas- 411 ticity: finite element solutions with a strain-difference-based formulation, J. 412 Math. Anal. Appl. 431 (2) (2015) 714-736. 